Trauma Berufskrankh 2004 5 [Suppl 1] : S149 DOI 10.1007/s10039-003-0815-z Online publiziert: 12.Dezember 2003

(c) Springer-Verlag 2003
D. Leuftink

Landesverband Südwestdeutschland der gewerblichen Berufsgenossenschaften, Heidelberg

\title{
Einführung in das Thema
}

er Diskussion zu dem bewusst in Frageform gekleideten Hauptthema „Akutbehandlung beendet - was nun?" stelle ich folgende Feststellungen voran:

1. Die Fragestellung ist nicht etwa Folge eines geringer gewordenen, möglicherweise gesetzlich beeinflussten Handlungsspielraums der Unfallversicherungsträger.

2. Es besteht keinesfalls eine gewisse Ratlosigkeit der handelnden Personen.

Nach wie vor ist die gesetzliche Unfallversicherung für die umfassende Rehabilitation ihrer Versicherten entsprechend dem Kriterium „Mit allen geeigneten Mitteln“ zuständig. Traditionell hat die Übungsbehandlung in diesem berufsgenossenschaftlichen Geschehensablauf einen hohen Stellenwert. Wenn wir uns dennoch mit dieser Thematik und der damit einhergehenden Schnittstellenproblematik beschäftigen, so scheint einerseits grundsätzlicher Regelungsbedarf gegeben. Es entspricht andererseits aber zudem unserer eigenen Handlungsphilosophie, auch oder gerade in Zeiten, in denen viele Sozialleistungen überhaupt in Frage gestellt werden, die eigene Arbeit auf den Prüfstand zu stellen. Gegebenenfalls muss nach Wegen gesucht werden, um den berufsgenossenschaftlichen Qualitätsstandard erhalten zu können. Mein persönlicher Optimismus, auch bei geänderten oder sich durch geringer werdende finanzielle Ressourcen möglicherweise noch weiter negativ verändernder Rahmenbedingungen handlungsfähig zu sein, ist begründet. Als Unfallversicherungsträger können wir nämlich verfahrensmäßig vieles tun und Einfluss nehmen, um damit bei gesamtheitlicher Betrachtungsweise des Rehabilitationsgeschehens den scheinbar konträren Gesichtspunkten von Humanität und Ökonomie entsprechen zu können.
Rund 200.000 stationäre Fälle bundesweit, davon etwa 30.000 im Bereich des Landesverbands Südwestdeutschland zeigen die rein statistische Dimension eines Themas auf, bei dem wir uns nach Abschluss der Akutbehandlung in der Tat die Frage stellen müssen, was nun geschehen soll. Regelmäßig wird es darum gehen, Maßnahmen der während der stationären Therapie begonnenen Übungsbehandlung fortzuführen oder erstmals einzuleiten. Aus unserem Verfahren des abgestuften Systems der Übungsbehandlung ist das nach Entlassung des Versicherten aus der Akutbehandlung individuell richtige Verfahren auszuwählen. Dafür kommen in Betracht:

1. die gezielte Physiotherapie als Standardtherapie

2. Maßnahmen der EAP als Komplextherapie oder

3. die BGSW, wenn die Übungsbehandlung nur unter stationären Bedingungen stattfinden kann.

Die Grenzen zwischen den einzelnen Verfahren mögen fließend sein und eine immer zutreffende Verordnung durch den Arzt erschweren; es muss aber dennoch möglich sein, unter Abwägung aller Einzelumstände die zutreffenden Maßnahmen zu veranlassen und aufwändigere und finanziell teurere Maßnahmen nur einzuleiten, wenn der gleiche Rehabilitationserfolg nicht auch durch „einfachere“ Maßnahmen der Übungsbehandlung erzielt werden kann. Im Übrigen: Ein Wechsel der Verfahren von der Standardtherapie (Basistherapie) bis hin zur BGSW ist - auf den Verletzungsfolgenzustand im konkreten Einzelfall abgestellt - ebenso möglich wie der umgekehrte Weg.

Eine zutreffende Antwort auf die Frage des weiteren Vorgehens im konkreten Einzelfall muss aber nicht nur fachlich fundiert sein; sie bedarf vielmehr, und zwar noch mehr als bisher, auch einer schnellen Entscheidung. Bekanntlich ist seit Jahren eine rückläufige stationäre Verweildauer zu beobachten. Diese wird jetzt sogar noch durch das neue Fallpauschalengesetz mit den DRG forciert. Dadurch verringert sich bei den Ärzten, aber insbesondere auch bei den berufsgenossenschaftlichen Verwaltungen die Reaktionszeit. Der zeitliche Rahmen zwischen dem Unfall und dessen Meldung beim Unfallversicherungsträger sowie dem Beginn notwendiger weiterer Rehabilitationsmaßnahmen wird nämlich immer enger. Verfrühte Entlassungen und/oder theoretisch mögliche Leistungsausdünnungen sind 2 Gründe, die zu raschem Handeln zwingen. Hinzu kommt, dass unsere Verfahren in Teilbereichen durch die verstrichene Zeit im Behandlungsablauf etwas „verwässert" wurden, indem z. B. Verletzte erst oder noch nach Monaten einer zu diesem Zeitpunkt medizinisch nicht indizierten BGSW-Maßnahme zugeführt wurden. Erinnern wir uns in diesem $\mathrm{Zu}$ sammenhang, dass BGSW „Berufsgenossenschaftliche Stationäre Weiterbehandlung" heißt, also eine Maßnahme ist, die grundsätzlich in zeitlichem Zusammenhang mit der Akutbehandlung steht.

Vor diesem Hintergrund werden wir nunmehr weitere Hinweise und Empfehlungen aus der Praxis erhalten.

Die heutige Veranstaltung kann damit auch den Blick dafür öffnen, wie sich die Qualität unserer Maßnahmen erhalten und wie sich die durch Akut- und Rehabilitationsbehandlung ergebende Schnittstellenproblematik verbessern lässt.

\section{Korrespondierender Autor D. Leuftink}

Landesverband Südwestdeutschland der gewerblichen Berufsgenossenschaften, Kurfürstenanlage 62,69115 Heidelberg E-Mail: service@heidelberg.lvbg.de 